\title{
Repetition rate after non-fatal self-poisoning in Sri Lanka: a one year prospective longitudinal study
}

\author{
T Rajapakse ${ }^{1,2}$, K M Griffiths ${ }^{2}$, S Cotton ${ }^{3}$, H Christensen ${ }^{4}$ \\ (Index words: non-fatal self-poisoning, repetition)
}

\begin{abstract}
Introduction Attempted or non-fatal self-poisoning is an important public health problem in Sri Lanka. Current evidence from Sri Lanka suggests that this phenomenon is more common among young people, and females, and is associated with a recent interpersonal conflict. International studies indicate that recent non-fatal selfharm is associated with an increased risk of repetition and completed suicide. Prospective follow-up data regarding rates of repetition of self-harm in Sri Lanka is limited.
\end{abstract}

Objectives The aim of this study was to describe the rate of repetition, and rate of suicidal ideation, at one-year follow up among those who have survived an act of selfpoisoning.

Methods Participants who presented to the toxicology unit, Teaching Hospital Peradeniya over a 14-month period, for medical management of non-fatal self-poisoning, were contacted by telephone one-year following the initial presentation.

Results A total of 949 persons were included in the study, of which $35.3 \% \quad(n=335)$ were contactable at one-year follow-up. The rate of repetition of self-harm after one year was $2.5 \%$ and $2.7 \%$ of participants had suicidal ideation at one-year follow-up.

Conclusions The rate of repetition of self-harm in Sri Lanka is lower than the rate reported in the West $(15 \%)$.

Ceylon Medical Journal 2016; 61: 154-158

http://doi.org/10.4038/cmj.v61i4.8380

\section{Introduction}

Non-fatal or attempted self-poisoning is an important public health problem in Sri Lanka with a reported rate of 321 per 100000 hospital admissions for self-poisoning in 2007 [1]. Since the mid-1990s, hospital admissions for non-fatal self-poisoning in Sri Lanka have increased [1]. Data from international studies indicate that recent non-fatal self-harm is associated with an increased risk of repetition and completed suicide [2]. It has been estimated that one in 25 self-harm patients will subsequently die by suicide in the 10 years after their index presentation [3]. Sri Lanka has an increasing rate of non-fatal self-harm by self-poisoning, but data on repetition rates are limited [4]. The SUPRE-MISS longitudinal follow-up study [5] reported that the repetition rate at 18 months in Sri Lanka was $4 \%$ (treatment as usual group) - substantially lower than the estimated one year repetition rate of $16 \%$ reported in the West $[3,6]$. In contrast, the rate of previous selfharm in those who have died by suicide in Sri Lanka has been reported to be as high as $26 \%[7,8]$.

The aim of this study was to describe the rate of repetition at one year follow up of those who have survived an act of self-poisoning. The specific objectives were to describe the rate of repeated suicide attempts (by any method) during the one year following the index non-fatal self-poisoning attempt, and the rates of suicidal ideation at one-year follow-up.

\section{Methods}

For purposes of this study, non-fatal self-poisoning was defined as intentional ingestion of a toxic substance or of a medication in excess of its prescribed dosage, with a non-fatal outcome; the term repetition was defined

\footnotetext{
${ }^{1}$ Faculty of Medicine, University of Peradeniya, Sri Lanka, ${ }^{2}$ National Institute for Mental Health Research, Australian National University, ${ }^{3}$ Centre for Youth Mental Health, University of Melbourne and ${ }^{4}$ Black Dog Institute, University of New South Wales, Australia.
}

Correspondence: TR, e-mail: <gemba471@gmail.com>. Received 18 March and revised version accepted 31 May 2016. 
as subsequent attempts of self-harm, by any method, during the one-year following the index act of non-fatal self-poisoning.

All persons who were admitted to the Toxicology Unit, Teaching Hospital Peradeniya, over a consecutive 14-month period starting from February 2012, were invited to participate in a cross-sectional survey for assessment of psychiatric morbidity, and were also followed up one year later. The baseline cross-sectional survey included a total of 949 participants. Details of the methodology and results of the baseline cross-sectional survey have been published elsewhere $[9,10]$, and will not be discussed further in this paper.

All participants of the baseline cross-sectional survey, which was conducted over 14 months, who had provided telephone numbers for follow-up contact ( $n=664$ ), were included for follow-up at one year. The follow-up involved a semi-structured telephone interview designed to determine suicidal ideation levels at 12 months, and the number of study participants who had repeated a suicide attempt (by any means) during the 12 months after the index non-fatal poisoning.

One year after the initial episode of non-fatal selfpoisoning, the participants were contacted via telephone. After the identity of the participant was confirmed as correct (via confirmation of his/her name), all those who gave verbal informed consent for the conversation were interviewed for about 10 minutes on the telephone. The participant was asked about the history of any repeat suicide attempts (by any method) during the one year period following the index self-poisoning attempt. Open ended queries were used to explore this theme. If there was a history of suicide attempts reported during this period, further details were elicited including the number of attempts, the timing of the attempts (relative to the index self-poisoning attempt) and the nature of the attempt. The presence of suicidal ideation at the time of the telephone interview and up to one week previously was explored via direct query.

If after several attempts, a participant could not be contacted on the telephone at the one year end point, the researcher attempted to contact the carer, friend or relative identified for that participant, during the index assessment. When contacting the friend or relative, the researcher introduced herself briefly, and inquired for contact details of the participant, while taking care to maintain participant confidentiality regarding the self-poisoning attempt. If the friend or relative was able to give new contact details for the participant, efforts were made to contact the participant again, and if this too failed, it was noted that the participant was lost to contact.

Approval for the study was obtained from the Ethics Review Committee Faculty of Medicine, University of Peradeniya, Sri Lanka, and the Human Research Ethics Committee of the Australian National University. Descriptive statistics and data analyses were undertaken using IBM ${ }^{\circledR}$ SPSS $^{\circledR}$ Statistics Version 21.0.

\section{Results}

A total of 1334 persons met eligibility criteria to be included in the baseline cross sectional survey, of whom $9.1 \%(n=121)$ refused consent, and $19.8 \%(n=264)$ could not be included because they either left hospital before the baseline survey could be conducted, or they were in hospital but were too unwell to participate. Thus a total of 949 persons participated in the baseline cross-sectional study, of whom $30.0 \%(n=285)$ did not provide telephone contact details and therefore could not be contacted at one year follow-up. Among those who provided telephone details $(n=664)$, in $49.5 \%(n=329)$ the telephone number given was no longer working or no longer being used by the participant at one year follow up. Thus, a total of $35.3 \%(n=335)$ of the participants of the baseline crosssectional survey were available for follow up at one year.

A comparison of the participants who were followed up and the participants who were not available for follow up is shown in Table 1 (based on data obtained during the baseline cross-sectional survey). The two groups were similar in most respects (Table 1). There were no significant differences between the two groups in age, gender, or substances ingested, rates of depression, alcohol use disorders, anxiety, suicidal intent, and past suicide attempts at baseline. The two groups differed in one respect - those lost to follow up were significantly more likely to have known a friend or relation who had also self-poisoned $\left(\chi^{2}(1)=12.8, p<0.001\right)$.

The repetition rates and suicidal ideation for participants who were followed up at one year are shown in table 2. Of those who could be contacted, 2.7\% $(n=9$, 95\% CI 1.67-3.73) had repeated an attempt of self-harm at one year follow up, and $2.5 \%(n=8,95 \%$ CI 1.51-3.49) reported suicidal ideation in the week prior to phone contact.

Visual inspection of percentages suggests that males had a higher rate of repetition compared to females, and that those aged between 25 and 34 years, and those aged $\geq 35$ years had a higher rate of repetition compared to those aged 14-24 years. With regards to suicidal ideation at one year, again visual examination of percentages suggests that males had higher rates compared to females, and those aged 25-34 years had higher rates compared to both younger and older age groups. However, the small numbers concerned precluded meaningful statistical analysis of possible associations between rates of repetition, suicidal ideation and variables such as age and gender. 
Table 1. Comparison of follow-up group and those who were not available for follow up (based on data obtained during the baseline cross-sectional survey)

\begin{tabular}{|c|c|c|c|c|}
\hline & & Follow-up group & Lost to follow up & p value \\
\hline Mean age & & 25.7 years & 25.4 years & .690 \\
\hline Proportion of females & $\%(\mathrm{n})$ & $55.8(187)$ & $55.9(343)$ & .990 \\
\hline \multicolumn{5}{|l|}{ Substance ingested } \\
\hline Medicinal overdose & $\%(n)$ & $61.0(202)$ & $55.0(335)$ & \\
\hline Pesticides & $\%(n)$ & $23.3(77)$ & $23.5(143)$ & .155 \\
\hline $\begin{array}{l}\text { Past suicide attempts } \\
\text { (prior to baseline presentation) }\end{array}$ & $\%(n)$ & $11.2(37)$ & $14.8(90)$ & .125 \\
\hline Depression (PDS) & $\%(\mathrm{n})$ & $50.4(137)$ & $53.0(260)$ & .494 \\
\hline \multicolumn{5}{|l|}{ Anxiety (GAD-7) } \\
\hline Moderate & $\%(n)$ & $23.3(59)$ & $18.6(47)$ & \\
\hline Severe & $\%(n)$ & $23.1(108)$ & $15.0(70)$ & .430 \\
\hline \multicolumn{5}{|l|}{ AUDIT } \\
\hline Hazardous drinking & $\%(\mathrm{n})$ & $8.4(28)$ & $9.4(57)$ & \\
\hline Alcohol use disorder & $\%(n)$ & $3.3(11)$ & $3.8(23)$ & .795 \\
\hline $\begin{array}{l}\text { Ingested alcohol prior to act } \\
\text { of non-fatal self-poisoning }\end{array}$ & $\%(n)$ & $9.5(31)$ & $11.1(66)$ & .449 \\
\hline BHS mean score & & 5.44 & 5.74 & .447 \\
\hline PSIS mean score & & 10.1 & 10.2 & .680 \\
\hline \multicolumn{5}{|l|}{ PSIS score (by category): } \\
\hline Low intent & $\%(n)$ & $7.2(24)$ & $4.4(27)$ & \\
\hline Medium intent & $\%(n)$ & $43.3(145)$ & $46.3(284)$ & \\
\hline High intent & $\%(n)$ & $49.6(166)$ & $49.3(303)$ & .173 \\
\hline $\begin{array}{l}\text { Has a past history of } \\
\text { outpatient psychiatric treatment }\end{array}$ & $\%(\mathrm{n})$ & $6.7(22)$ & $6.3(38)$ & .811 \\
\hline $\begin{array}{l}\text { Has a past history of inpatient } \\
\text { psychiatric treatment }\end{array}$ & $\%(\mathrm{n})$ & $2.9(9)$ & $4.2(22)$ & .345 \\
\hline $\begin{array}{l}\text { Knew a friend/ relation who } \\
\text { has self-poisoned in the past }\end{array}$ & $\%(n)$ & $12.3(38)$ & $22.2(126)$ & .001 \\
\hline
\end{tabular}

Notes for abbreviations

PDS: $\quad$ Peradeniya Depression Scale. Scoring: Total score $\square$ 10/25 is a positive screening for depression

GAD-7: 7-item anxiety scale. Scoring: 5-9 Mild, 10-14 Moderate, 15-21 severe anxiety

AUDIT: Alcohol Use Disorders Identification Test. Scoring: $\square 7=$ Hazardous drinking (HZD drinking), $\square 16=$ Alcohol use disorder (AUD)

BHS: $\quad$ Beck Hopelessness Scale

PSIS: $\quad$ Pierce Suicide Intent Scale. Total score range: 0-21. Scoring by category: low intent (total score 0-3), medium intent (total score 4-10) and high intent (total score $>10$ ).

Table 2. Repetition of suicidal attempts and presence of suicidal ideation, at one-year follow-up

\begin{tabular}{|c|c|c|c|c|c|c|c|}
\hline & \multirow{2}{*}{$\begin{array}{c}\%(n) \\
(\text { Total } n=335)\end{array}$} & \multirow{2}{*}{$\begin{array}{c}95 \% C I \\
\text { of } \%\end{array}$} & \multicolumn{2}{|c|}{ By gender } & \multicolumn{3}{|c|}{ By age } \\
\hline & & & $\begin{array}{c}\text { Male } \\
(n=148) \\
\%(n)\end{array}$ & $\begin{array}{c}\text { Female } \\
(n=187) \\
\%(n)\end{array}$ & $\begin{array}{c}14-24 y r s \\
(n=205) \\
\%(n)\end{array}$ & $\begin{array}{c}25-34 \text { yrs } \\
(n=81) \\
\%(n)\end{array}$ & $\begin{array}{c}\text { Over } 35 \text { yrs } \\
(n=49) \\
\%(n)\end{array}$ \\
\hline $\begin{array}{l}\text { Repetition at } \\
\text { one year }\end{array}$ & $2.7(9)$ & $1.67-3.73$ & $4.1(6)$ & $1.6(3)$ & $2.0(4)$ & $3.7(3)$ & $4.1(2)$ \\
\hline $\begin{array}{l}\text { Suicidal ideation at } \\
\text { one year }\end{array}$ & $2.5(8)$ & $1.51-3.49$ & $3.5(5)$ & $1.7(3)$ & $2.0(4)$ & $4.9(4)$ & 0 \\
\hline
\end{tabular}




\section{Discussion}

The repetition rate of $2.7 \%(n=9)$ at one year follow up is substantially lower than the rates of repetition reported in the West, where the estimated one year repetition rate of non-fatal self-harm has been reported to be $16.3 \%[2,3,6,11]$. A limitation of this study is that only $35.3 \%$ of the original sample was available for follow-up. Although (with one exception) there were no differences at index episode between those who were and were not available for follow-up, the group that was followed up may have been less likely to re-attempt. The fact that the followup group had provided a telephone contact, had stable contact details, and were willing be interviewed may indicate underlying differences from the group that was not available for follow-up, which may have influenced the findings. It is also possible that some of the group lost to follow-up were not available due to a completed suicide. On the other hand, lower rates of repetition in Asian countries compared to the West has been described previously, and it has been suggested that rates of repetition in Asia may be lower than rates reported in the West [3].

If repetition rates in Asian countries such as Sri Lanka are truly lower than rates reported from the West, there is a need to explore why. One explanation might be that there is higher case fatality associated with self-harm in Asian countries such as Sri Lanka, compared to the West, due to the lethality of substances ingested (i.e., pesticides) [12]. As a result those potential repeaters in the West may not survive their first self-harm attempt in Asia. Another possible explanation - that has also been proposed before is the prolonged hospital stay due to ingestion of toxic substances in Asian countries such as Sri Lanka, compared to the much shorter hospital stay associated with ingestion of less toxic medicinal overdoses, seen in the West [3, 13]. Since risk of repetition is highest during the early period following self-harm, it has been suggested that this prolonged hospital stay may inhibit further self-harm during this high risk period [3]. Another possibility is that the recollection of the adversities faced due to the index poisoning episode, including the hospital stay and treatment, would serve as a deterrent against further self-poisoning attempts. Thus, the difficulties associated with a hospital stay in a developing country setting may serve as a reminder and an inadvertent but potent preventive against future self-poisoning.

A final possible explanation for a low repetition rate of suicide attempts relates to the motivations underlying the initial non-fatal self-poisoning act, especially in young people and females in countries such as Sri Lanka. Non-fatal self-poisoning in Sri Lanka has been often described as being associated with a short duration of premeditation, and recent inter-personal conflict $[10,14]$. The act of self-poisoning in Sri Lanka among girls may represent a form of nonverbal dissent, in situations of interpersonal conflict where norms of obedience and respect constrain overt responses [15]. If the act of non-fatal self-poisoning resulted in attention being directed to that young female's dilemma (which she had been unable to articulate directly), and if this resulted in some sort of a resolution on part of that person's family, the risk or motivation for repetition may be low. This theory requires further exploration and evidence.

The main limitation of this study was that only $35.3 \%$ $(n=335)$ of those who participated in the baseline survey were available for follow-up contact at one year. As noted above, this may have influenced the findings, and low numbers also limited further analysis of the data. Further, the fact that $9.1 \%$ of the eligible sample refused consent to participate at baseline, and a further $19.8 \%$ could not be included due to having left the hospital/ having been too physically unwell to participate in the study is also a limitation, and may have influenced the findings. There was no data on how many amongst those not followed-up may have died by suicide; this too may have confounded the findings. Another limitation was that the follow-up interview was conducted over the phone rather than faceto-face, which may have influenced participant disclosure of attempts and suicidal ideation. Factors such as the wish to avoid another hospital admission, or stigma, may also have influenced participants to under-report suicidal attempts or suicidal ideation. However every effort was made to conduct the phone interviews in a confidential manner, at a time convenient to the participant, and a semi-structured questionnaire was used to ensure that all interviews were conducted in a uniform manner.

\section{Conclusions}

The rate of repetition at one year in this study is low, similar to other Asian studies, but in contrast to Western data [3]. While these findings may have been influenced by the low follow-up rate in this study, if confirmed, the apparent low repetition rate may have important implications for prevention strategies. In particular, the pattern reinforces the importance of implementing strategies at a primary preventive level since the potential impact of secondary prevention techniques may be limited by the low repetition rates.

\section{Acknowledgements}

The authors acknowledge the staff of the Toxicology Unit, Teaching Hospital Peradeniya, and the South Asian Clinical Toxicology Research Collaboration for their help. 


\section{Conflicts of interest}

There are no conflicts of interest.

\section{References}

1. De Silva V, Senanayake SM, Dias P, Hanwella R. From pesticides to medicinal drugs: time series analyses of methods of self-harm in Sri Lanka. Bull World Health Organ 2012; 90: 40-6.

2. Owens D. Fatal and non-fatal repetition of self-harm: Systematic review. Br J Psychiatry 2002; 181: 193-9.

3. Carroll R, Metcalfe C, Gunnell D. Hospital Presenting Self-Harm and Risk of Fatal and Non-Fatal Repetition: Systematic Review and Meta-Analysis. PLoS One 2014; 9: e89944.

4. Hanwella R, Senanayake SM, De Silva V. Geographical variation in admissions due to poisoning in Sri Lanka: a time series analysis. Ceylon Med J 2012; 57: 152-8.

5. Bertolote JM, Fleischmann A, De Leo D, et al. Repetition of suicide attempts: data from emergency care settings in five culturally different low- and middle-income countries participating in the WHO SUPRE-MISS Study. Crisis 2010; 31: 194-201.

6. Kapur N, Cooper J, King-Hele S, et al. The repetition of suicidal behavior: a multicenter cohort study. J Clin Psychiatry 2006; 67: 1599-609.
7. Samaraweera S, Sumathipala A, Siribaddana S, Sivayogan $\mathrm{S}$, Bhugra D. Completed suicide among Sinhalese in Sri Lanka: a psychological autopsy study. Suicide Life Threat Behav 2008; 38: 221-8.

8. Abeyasinghe R, Gunnell D. Psychological autopsy study of suicide in three rural and semi-rural districts of Sri Lanka. Soc Psychiatry Psychiatr Epidemiol 2008; 43: 280-5.

9. Rajapakse T, Griffiths KM, Christensen H, Cotton S. A comparison of non-fatal self-poisoning among males and females, in Sri Lanka. BMC Psychiatry 2014; 14: 221.

10. Rajapakse T, Griffiths KM, Christensen H, Cotton S. Nonfatal self-poisoning in Sri Lanka: associated triggers and motivations. BMC Public Health 2015; 13: 1-7.

11. Hawton K, Fagg J. Trends in deliberate self poisoning and self injury in Oxford, 1976-90. BMJ 1992; 304: 1409-11.

12. Eddleston M, Gunnell D, Karunaratne A, de Silva D, Sheriff MHR, Buckley NA. Epidemiology of intentional self-poisoning in rural Sri Lanka. Br J Psychiatry 2005; 187: 583-4.

13. Mohamed F, Perera A, Wijayaweera K, Kularatne K, Jayamanne S, Eddleston $\mathrm{M}$, et al. The prevalence of previous self-harm amongst self-poisoning patients in Sri Lanka. Soc Psychiat Epidemiol 2010; 7: 1-4.

14. Hettiarachchi J, Kodituwakku GC. Self poisoning in Sri Lanka: motivational aspects. Int J Soc Psychiatry 1989; 35: $204-8$.

15. Marecek J, Senadheera C. 'I drank it to put an end to me': Narrating girls' suicide and self-harm in Sri Lanka. Contrib Indian Sociol 2012; 46: 53-82. 